\title{
MULTIPLES OF WEIERSTRASS POINTS AS SPECIAL DIVISORS
}

\author{
R. F. LAX
}

ABSTRact. Complex spacss $W_{n}^{r}$ of Weierstrass points are isomorphic to the intersection, on the $n$th symmetric product of the universal curve over the Teichmüller space, of complex spaces $\mathcal{G}_{n}^{r}$ of special divisors with the diagonal $\Delta_{n}$ consisting of divisors which are multiples of a point. The tangent space at a point of this intersection is described and it is shown that $\mathcal{G}_{n}^{1}-\mathcal{G}_{n}^{2}$ and $\Delta_{n}$ intersect transversally.

Let $T=T_{g}$ denote the Teichmüller space for Teichmüller surfaces of genus $g>1$ and let $\pi: V \rightarrow T$ denote the universal curve of genus $g$. Denote by $V_{T}^{(n)}$ the $n$th symmetric product of $V$ over $T$. Let $\mathcal{G}_{n}^{r}$ denote the closed complex subspace of $V_{T}^{(n)}$ whose points are divisors of degree $n$ and projective dimension at least $r$ (see [3], [2]). We have proved

TheOREM 1 ([3]). Suppose $n \leqslant g$. Then $\mathcal{G}_{n}^{1}-\mathcal{G}_{n}^{2}$ is smooth of pure dimension $2 n+2 g-4$.

For $2 \leqslant n \leqslant g$, let $w_{n}^{r}$ denote the closed complex subspace of $V$ consisting of those $(t, P) \in V$ such that there are at least $r$ gaps less than or equal to $n$ in the Weierstrass gap sequence at $P$ on $V_{t}$. These spaces were introduced in [4] and, by employing methods similar to those used in the proof of Theorem 1, we proved

THEOREM 2 ([4]). For $2 \leqslant n \leqslant g, W_{n}^{1}-W_{n}^{2}$ is smooth of pure dimension $n+2 g$ -3 .

In this note, we describe the relationship between the $\mathcal{G}_{n}^{r}$ and the $\mathscr{W}_{n}^{r}$ and show how Theorem 2 may be derived in a direct fashion from Theorem 1.

Let $\Delta_{n}$ denote the image of $\delta_{n}: V \rightarrow V_{T}^{(n)}$, the closed immersion which takes a point $(t, P)$ to the point $(t, n P)$. The following proposition follows easily from the definitions.

Proposition 1. For $2<n<g$,

$$
\left.\delta_{n}\right|_{w_{n}^{r}}: w_{n}^{r} \stackrel{\approx}{\rightarrow} \mathcal{G}_{n}^{r} \cap \Delta_{n} .
$$

We now explicitly consider the intersection of $\mathcal{G}_{n}^{r}$ and $\Delta_{n}$. Suppose $(t, n P) \in$ $\mathcal{G}_{n}^{r} \cap \Delta_{n}$. Put $X=V_{t}$. Let $z$ be a local coordinate on $X$ centered at $P$ and let $z_{1}, \ldots, z_{n}$ denote $n$ copies of $z$. Let $\sigma_{1}, \ldots, \sigma_{n}$ denote the $n$ elementary symmetric functions in $z_{1}, \ldots, z_{n}$. Let $c_{1}, \ldots, c_{3 g-3}$ denote Patt's local coordinates on $T$

Received by the editors May 8, 1980.

1980 Mathematics Subject Classification. Primary 32G15, 14 H15.

Key words and phrases. Complex space, Weierstrass point, special divisor, Teichmüller space, universal curve. 
centered at $t$ (see [8]). Then $c_{1}, \ldots, c_{3 g-3}, \sigma_{1}, \ldots, \sigma_{n}$ are local coordinates on $V_{T}^{(n)}$ centered at $(t, n P)$. Put

$Z=$ tangent space to $V_{T}^{(n)}$ at $(t, n P)$;

$Z_{1}=$ tangent space to $\mathcal{G}_{n}^{r}$ at $(t, n P)$;

$Z_{2}=$ tangent space to $\Delta_{n}$ at $(t, n P)$.

We describe coordinates for $Z$. Suppose $\xi \in Z$. We may view $\xi$ as a $\mathbf{C}$-homomorphism of local rings

$$
\xi: \Theta_{V(n),(t, n P)} \rightarrow \mathbf{C}[\varepsilon] /\left(\varepsilon^{2}\right)
$$

(cf. [6, p. 332]). Then $\xi$ is determined by its values on a set of local parameters of $\theta_{V_{f}^{(n)},(t, n P)}$. So, if $\xi\left(c_{m}\right)=b_{m} \varepsilon, m=1, \ldots, 3 g-3$, and $\xi\left(\sigma_{i}\right)=u_{i} \varepsilon, i=1, \ldots, n$, then $\left(u_{1}, \ldots, u_{n}, b_{1}, \ldots, b_{3 g-3}\right)$ serve as coordinates for $Z$.

Proposition 2. $\xi=\left(u_{1}, \ldots, u_{n}, b_{1}, \ldots, b_{3 g-3}\right)$ is in $Z_{2}$ if and only if $u_{2}=u_{3}$ $=\cdots=u_{n}=0$.

Proof. Suppose $\left(t_{1}, Q_{1}+\ldots+Q_{n}\right) \in V_{T}^{(n)}$ is a point near $(t, n P)$. Then $\left(t_{1}, Q_{1}\right.$ $\left.+\cdots+Q_{n}\right) \in \Delta_{n} \Leftrightarrow z\left(Q_{1}\right)=\cdots=z\left(Q_{n}\right)=z_{0} \Leftrightarrow z_{0}$ is an $n$-fold root of

$$
\begin{aligned}
F(Y)= & \prod_{i=1}^{n}\left(Y-z\left(Q_{i}\right)\right)=Y^{n}-\sigma_{1}\left(z\left(Q_{1}\right), \ldots, z\left(Q_{n}\right)\right) Y^{n-1} \\
& +\cdots+(-1)^{n} \sigma_{n}\left(z\left(Q_{1}\right), \ldots, z\left(Q_{n}\right)\right) \\
\Leftrightarrow & F\left(z_{0}\right)=F^{\prime}\left(z_{0}\right)=\cdots=F^{(n-1)}\left(z_{0}\right)=0 \\
\Leftrightarrow & \sigma_{k}\left(z\left(Q_{1}\right), \ldots, z\left(Q_{n}\right)\right)=\left(\begin{array}{l}
n \\
k
\end{array}\right)\left[\sigma_{1}\left(z\left(Q_{1}\right), \ldots, z\left(Q_{n}\right)\right)\right]^{k} / n^{k}
\end{aligned}
$$

for $k=2,3, \ldots, n$,

and $\sigma_{1}\left(z\left(Q_{1}\right), \ldots, z\left(Q_{n}\right)\right)=n z_{0}$.

So, near $(t, n P), \Delta_{n}$ is defined by the equations $\left\{\sigma_{k}=\left(\begin{array}{c}n \\ k\end{array}\right) \sigma_{1}^{k} / n^{k}\right\}, k=2, \ldots, n$. Thus $\xi$ is tangent to $\Delta_{n}$ at $(t, n P)$ if and only if $\xi\left(\sigma_{k}\right)=0$ for $k=2,3, \ldots, n$.

We next recall the description of $Z_{1}$, which was given in [3]. Let $1, \gamma_{2}, \ldots, \gamma_{g}$ denote the Weierstrass gaps at $P \in X$. Choose a basis of holomorphic 1-forms $d \zeta_{1}, \ldots, d \zeta_{g}$ on $X$ such that $\operatorname{ord}_{P} d \zeta_{j}=\gamma_{j}-1$. Write

$$
d \zeta_{j}=\sum_{i=0}^{\infty} a_{i, j} z^{i} d z .
$$

For details concerning the following result, we refer the reader to [3].

Proposition 3. Suppose $n<g$ and $\xi \in Z$. Then $\xi \in Z_{1}$ if and only if all minors of order $n-r+1$ of the matrix

$$
\mathfrak{R}=\left[\begin{array}{l:l}
(-1)^{i} a_{i, j} & \varepsilon\left[\sum_{l=1}^{n}(-1)^{i+l-1} a_{i+l} u_{l}+\sum_{m=1}^{3 g-3} \tau_{P, i}^{\prime}\left(Q_{m}\right) \zeta_{j}^{\prime}\left(Q_{m}\right) b_{m}\right] \\
i=0, \ldots, n-1 & i=0, \ldots, n-1 \\
j=1, \ldots, n-r & j=n-r+1, \ldots, g
\end{array}\right]
$$

vanish, where $\tau_{P, k}$ is an elementary integral of the second kind on $X$ with pole of order $k+1$ at $P$ and where $\left(Q_{1}, \ldots, Q_{3 g-3}\right)$ is any point chosen from an open subset of $X^{38-3}$. 
Now, suppose $(t, n P) \in \mathcal{G}_{n}^{r}-\mathcal{G}_{n}^{r+1}, n<g$. Then $\mathfrak{T}$ will have a nonzero minor of order $n-r$, call it $\mu$, and in order that all minors of order $n-r+1$ of $\pi$ vanish, it is sufficent that those minors of order $n-r+1$ which contain $\mu$ should vanish. This gives rise to $r(g-n+r)$ linear equations $\left\{E_{k}\right\}$ in $u_{1}, \ldots, u_{n}$, $b_{1}, \ldots, b_{3 g-3}$. These equations are of the form

$$
E_{k}: \sum_{l=1}^{n} e_{k, l} u_{l}+\sum_{m=1}^{3 g-3} \alpha_{k}\left(Q_{m}\right) b_{m}=0
$$

where the $\alpha_{k}$ are (not necessarily finite) quadratic differentials on $X$. (The $\alpha_{k}$ arise from the products $d \tau_{P, i} d \zeta_{j}$ which appear in $\Re$-see [3].)

THEOREM 3. Suppose $n \leqslant g, r(g-n+r)<3 g-3$, and $(t, n P) \in \mathcal{G}_{n}^{r}-\mathcal{G}_{n}^{r+1}$. If the above $\alpha_{k}, k=1, \ldots, r(g-n+r)$, are linearly independent quadratic differentials, then:

1) $\operatorname{dim} Z_{1}=3 g-3+(r+1)(n-r)-r g+r$ and $\mathcal{G}_{n}$ is smooth at $(t, n P)$.

2) $\operatorname{dim} Z_{1} \cap Z_{2}=3 g-2-r(g-n+r)$ and $\mathcal{G}_{n}^{r}$ and $\Delta_{n}$ intersect transversally at $(t, n P)$.

Proof. One may show, as in [3], that if the $\alpha_{k}$ are linearly independent, then since $\left(Q_{1}, \ldots, Q_{3 g-3}\right)$ is any point from an open subset of $X^{3 g-3}$, the matrix $\left[\alpha_{k}\left(Q_{m}\right)\right], k=1, \ldots, r(g-n+r)$ and $m=1, \ldots, 3 g-3$, will have maximum rank. It then follows that the systems of equations which define $Z_{1}$ and $Z_{1} \cap Z_{2}$ will have maximum rank, establishing the theorem.

We showed in [3] that at least $g-n+r$ of the $\alpha_{k}$ are linearly independent. In particular, if $r=1$, then all the $\alpha_{k}$ are linearly independent. As a consequence we have

THEOREM 4. Suppose $(t, n P) \in \mathcal{G}_{n}^{r}-\mathcal{G}_{n}^{r+1}, n<g$. Then

(1) $\operatorname{dim} Z_{1} \leqslant 2 g+2 n-r-3$; in particular, if $r=1$, then $\operatorname{dim} Z_{1}=2 g+2 n-$ 4 and $\mathcal{G}_{n}^{1}$ is smooth at $(t, n P)$.

(2) $\operatorname{dim} Z_{1} \cap Z_{2} \leqslant 2 g+n-r-2$; in particular, if $r=1$, then $\operatorname{dim} Z_{1} \cap Z_{2}=$ $2 g+n-3$ and $\mathcal{G}_{n}^{1}$ and $\Delta_{n}$ intersect transversally at $(t, n P)$.

COROllary. For $n \leqslant g$,

(1) $\operatorname{dim} w_{n}^{r} \leqslant 2 g+n-r-2$;

(2) $\mho_{n}^{1}-\mho_{n}^{2}$ is smooth of pure dimension $2 g+n-3$.

REMARKs. (1) The smoothness of $\mathcal{G}_{n}^{1}-\mathcal{G}_{n}^{2}$ has also recently been demonstrated by Arbarello-Cornalba [1] and Namba [7].

(2) Arbarello-Cornalba [1] have shown that $\mathcal{G}_{n}^{2}-\mathcal{G}_{n}^{3}$ is smooth, but it does not necessarily follow that the $\alpha_{k}$ are then linearly independent or that this space intersects $\Delta_{n}$ transversally.

(3) In [5], we defined $W_{n}^{r}$ for $n>g$. The points of this space are those $(t, P) \in V$ such that there are at least $r$ gaps greater than $n$ in the gap sequence at $P \in V_{t}$. We showed that for $n>g$, $W_{n}^{1}-\mathcal{W}_{n}^{2}$ is smooth of pure dimension $4 g-n-3$. This result can also be obtained as above by considering the intersection of $\mathcal{G}_{n}^{r}$ and $\Delta_{n}$ for $n>g$, but we note that, by our definition of $\mathcal{W}_{n}^{r}$, for $n>g, W_{n}^{r}=\delta_{n}^{-1}\left(\mathcal{G}_{n}^{n-g+r}\right)$. 


\section{REFERENCES}

1. E. Arbarello and M. Cornalba, Su una congettura di Petri, Comment Math. Helv. (to appear).

2. R. C. Gunning, Lectures on Riemann surfaces: Jacobi varieties, Princeton Univ. Press, Princeton, N. J., 1972.

3. R. F. Lax, On the dimension of varieties of special divisors, Trans. Amer. Math. Soc. 203 (1975), 141-159.

4. Weierstrass points of the universal curve, Math. Ann. 216 (1975), 35-42.

5. Gap sequences and moduli in genus 4, Math. Z. 175 (1980), 67-75.

6. D. Mumford, Introduction to algebraic geometry (preliminary version of first 3 chapters), Harvard notes.

7. M. Namba, Families of meromorphic functions on compact Riemann surfaces, Lecture Notes in Math., vol. 767, Springer-Verlag, Berlin, 1979.

8. C. Patt, Variations of Teichmüller and Torelli surfaces, J. Analyse Math. 11 (1963), 221-247.

Department of MATHEMatics, Northeastern University, Boston, MASSAChusetTs 02115

Department of Mathematics, Louisiana State University, Baton Rouge, Louisiana (Curtent address) 